\title{
Using PRAAT for Analysing Segmental Features of Speech Produced by the Students of English Study Program of Universitas Brawijaya
}

\author{
Istiqomah Wulandari \\ Iis Nur Rodliyah \\ Fatimah \\ English Study Program of Universitas Brawijaya
}

\begin{abstract}
Making improvements of the speech feature is possible to do because pronunciation is teachable. Some experts in pronunciation teaching have suggested more comprehensive and contemporary teaching methods and techniques. In narrower context to English Study Program some seemingly cases are found in depicting the condition of how the last semester students who attend thesis seminar are tend to have difficulty in their Pronunciation. Many of them are still struggling in articulating the correct English pronunciation. The problematic speech features are targeted to the segmental part, such as the accuracy of vowel sounds production. For this particular research, the researchers want to investigate the similar problematic features of pronunciation of the second semester students of English Study Program of Universitas Brawijaya and later will try to implement some teaching pronunciation techniques to modify their pronunciation production. For this particular research employs a qualitative research design. The data were analysed into narrative description, interpretation and textual. The participants were asked to read aloud the given 14 minimal pair words list. The recorded data were saved as wave files (.wav) to analyze them by using PRAAT to visual display of the students' First Formant (F1) and Second Formant (F2). Then, these visual displays were compared to those of native speakers in PRAAT window. The result of this research reveals that due to the lack of how to disambiguate the correct pronunciation of vowel sounds within the given words the participant tend to produce inaccurate pronunciation. Which are reflected by the result of the spectrum score range: F1 and F2. Some proposed techniques of teaching segmental were drilling, teaching minimal pairs, chanting with jazz chant and rhyming with tongue twisters, to modify learners segmental features. As the suggestion all targeted parties (students, Lectures and Authority) should be willing to do what are necessary to maintain the success of the teaching and learning pronunciation in Study program of English.
\end{abstract}

Key words: student' spoken feature, segment

\section{Background of the Study}

There are some suggestions that have been initiated for pronunciation teaching in the classroom by language expertss. One of which is pronunciation should be taught in integrated way along with other language skills especially listening and speaking to prepare the language competence of the students. But somehow the practise of teaching pronunciation cannot go beyond the drilling and repeating unit of sounds (vowels and consonants), in which materials are taken from various English pronunciation websites. Firstly, students listen to the sound and eventually will be able to produce their own sounds. It seems that the two main goals of this learning pronunciation are to recognise and reproduce the sounds. The teaching instruction involves the use of a recorder that goes along with the handouts. Later, there is a change in the way how pronunciation is supposed to be taught. Indonesian English teachers have read many books about reconstruction of the traditional way of teaching instruction for pronunciation and they regard pronunciation is not merely learning individual vowels or consonants but rather as a whole unit of sounds that include word stress, sentence stress and intonation, which are supposed to be taught alongside with other main language skills, especially speaking and listening (Matthews, 1994). Consequently, the teachers have been 
"struggling" to teach pronunciation within the context of speaking and listening learning activities.

In addition to learning isolated vowels and consonants, students also learn the intonation and stress patterns from the listening activities taken from various selected students' workbooks such as: Active Listening (CUP), New Interchange (CUP), Cutting Edge (Longman), New Headaway (OUP), Quest (McGraw- Hill), and Lecture Ready (OUP). Apparently some of English teachers might then start teaching intonation and stress pattern based on what those workbooks suggest, and not to mention with the little knowledge on using the appropriate techniques. A sample research has been conducted by Pardede, P. (2007) on an Error Analysis on the Production of English Fricatives by the Freshmen of the English Department of FKIP-UKI Jakarta. This research can be used as the theoretical ground for the participant speech feature analysis. The research finding revealed that the Indonesian sound system affects to some extent for Indonesian student who learn the English language. The English fricative sound $/ \delta /, / \theta /$ are commonly difficult to pronounce by Indonesian students and they tend to substitute the sound to alveolar stop $/ \mathrm{t} /$ and $/ \mathrm{d} /$. For example for the word that $/ ð æ t /---/ d æ t /$, and the word thin /' $\theta \mathrm{In} /---$ /tin/. The possible reason why the substitution happens because Indonesian language does not have fricative sound $/ \delta /, / \theta /$ features for its consonant. Even if we have some words using this particular feature the words are usually borrowed from foreign language. For example the word dholim and adhan are borrowed from Arabic language. The other problematic English pronunciation for Indonesian students is the different phonotactic patterns of English language from that of Indonesian. For example Indonesian tends to insert a vowel to consonant cluster in the word speaking for instance. Indonesian student tends to say /sopikin/ instead of /spikin/. Although some Indonesian words consist consonant cluster they are usually borrowed words from foreign language such as dholim, adhan, ikhtiar, which are from Arabic language adaptation and the word kwalitas is borrowed from English. There are many local dialects with its various phonotactic patterns that might affect the Indonesian students in learning English pronunciation. More over the distinctive inventory system of sounds are also might be influential in learning pronunciation. For example the difficulty of Indonesian to pronounce the aspirated English consonant $/ \mathrm{p} /, / \mathrm{t} /, / \mathrm{k} /$ since all Indonesian consonant are not aspirated (Pardede, 2007). The research conducted by Pardede (2007) seems to support the essential features of Native Speaker (NNS) communication by Jenkins (2007) which might the result of different phonotactic patterns and its inventory systems.

Obviously, pronunciation teaching has become an interesting and challenging issue to support the success of second language learning. Making improvements of the speech feature is possible to do because pronunciation is teachable. Some experts in pronunciation teaching have suggested more comprehensive and contemporary teaching methods and techniques that include multi-modalities features (Acton, 2011; Dickerson 2010; and Gilbert, 2008) to counter the previous traditional techniques of drilling and repeating the sounds. In this research, the researchers will try to relate those current issues on the teaching pronunciation in Indonesia, and try to see how those issues are addressed to those theories and thoughts. Later some samples of pronunciation teaching techniques will be introduced to modify common problematic elements of speech features produced by English language learners. Those problematic speech features are ranging from segmental to prosodic features, such as: phonological process of segmental features, intonation pattern, tone unit and fluency. In smaller context base on day to day teaching experiences and attending students thesis seminars in English Study Program. There some seemingly cases are found in depicting the condition of how students even for the last semester who attend thesis seminar are tend to have difficulty in their Pronunciation. Many of 
them are still struggling in articulating the correct English pronunciation. Thus this phenomenon is interestingly motivating the researchers to conduct research under the theme of analysing students' spoken features, in an expectation to prepare the new semester students to have a correct pronunciation starting in very early stage. For this particular research, the researchers want to investigate the similar problematic features of pronunciation of the second semester students of English Study Program of Department of Universitas Brawijaya and later will try to implement some teaching pronunciation techniques to modify their pronunciation production.

\section{Methodology}

This research aims to understand the "complexity" of the data (Kervin et al, 2006, p.35) through interpretation and reflection to establish the meaning (Cliff, 2012). Moreover, this research concerns with using data sources to collect "thick description," (Kervin et al, 2006, p. 84), thus the data are analysed into narrative description, interpretation and textual (Cliff, 2012). The data for this research were collected form interview and record. The participants were asked to read aloud the given minimal pairs words list and were recorded. Later the recorded data were analysed based on their spoken features to find out which the problematic features are.

The participants of this research were purposely selected "because of their key involvement within the social setting and their ability to tell the researcher what they observe, think and feel," (Kervin et al, 2006, p.106). It is well known as a purposive sampling (Cliff, 2012). Purposive sampling is conducted to meet the aim of qualitative research which is describing occurrences in a particular setting. In recruiting the sample of qualitative researcher might "use his or her judgment as to which segments should be included," (Charles \& Mertler, 2002, p.141), thus the participants chosen are suitable for the research needs. For this particular research the purposive sampling is the $10 \%$ taken out of the population of the second semester students of study program of English of
Universitas Brawijaya which are approximately over 100 . The sample were categorized as quota sample, means the number of participant has been limited only $10 \%$ out the population due to the limitation of time and capability of the researchers in collecting and analaysing the sample. This conditition is considered appropriate and in accordance to what Arikunto's (2010) argument that for descriptive research taking $10 \%$ sample out of the population is allowed. The choice is underlined by an assumption of being the freshmen students; the chance of having mispronounced the English sounds is relatively understandable. Accordingly the modification of their pronunciation production is needed to improve their English pronunciation.

The data of this research were analysed as follows: the recorded spoken of scripted minimal pairs were saved as wave files (.wav) to analyze them by using PRAAT software. The students' voices were put in this program. There was visual display of the students' speech. Then, these visual displays were compared to those of native speakers in PRAAT window in form of spectrogram. The data were presented in the form of Hertz $(\mathrm{Hz})$ tabulation of the First Formant (F1) and the Second Formant (F2). This step is done in order to determine whether the students have produced the utterances in a target-like manner. These steps are done to answer the first research problem about the problematic features in pronunciation produced by the second semester students of English Study of English Program of Universitas Brawijaya. Finally, the researchers formulated the data analysis result; the researchers can have information upon how the participant of the study is going to be treated. A particular treatment is given to modify their pronunciation production by implementing some selected techniques in learning pronunciation.

\section{Discussion}

The discussion section highlights interesting phenomena from the data analysis and concerning the students' problematic segmental features in pronouncing the 14 minimal pairs words 
list. Later it will be related to the issues of intelligibility.

\subsection{Analysis of speech features}

As mentioned earlier, this section displays and analyses the speech features of the second semester students of the English Study Program. The speech features are in a tabulation result of the graphic by using PRAAT software. The tabulation result of the graphic form of both a native speaker of English and students speech features are displayed in the following

tables.

Table 4.1 the tabulation result of the native speaker's speech features

\begin{tabular}{|l|l|l|l|}
\hline No & \multicolumn{1}{|c|}{ Word } & First Formant (F1) & Second Formant (F2) \\
\hline 1 & Beat & $289.95 \mathrm{~Hz}$ & $2138.92 \mathrm{~Hz}$ \\
\hline 2 & Beard & $348.86 \mathrm{~Hz}$ & $1844.29 \mathrm{~Hz}$ \\
\hline 3 & Bait & $413.11 \mathrm{~Hz}$ & $2023.29 \mathrm{~Hz}$ \\
\hline 4 & Bed & $481.27 \mathrm{~Hz}$ & $1701.05 \mathrm{~Hz}$ \\
\hline 5 & Bad & $654.13 \mathrm{~Hz}$ & $1462.38 \mathrm{~Hz}$ \\
\hline 6 & Body & $537.39 \mathrm{~Hz}$ & $1626.92 \mathrm{~Hz}$ \\
\hline 7 & Bought & $597.49 \mathrm{~Hz}$ & $1126.54 \mathrm{~Hz}$ \\
\hline 8 & Buddhist & $811.43 \mathrm{~Hz}$ & $2114.70 \mathrm{~Hz}$ \\
\hline 9 & Boat & $448.63 \mathrm{~Hz}$ & $1367.80 \mathrm{~Hz}$ \\
\hline 10 & Boot & $352.38 \mathrm{~Hz}$ & $1499.64 \mathrm{~Hz}$ \\
\hline 11 & Bud & $546.17 \mathrm{~Hz}$ & $1453.14 \mathrm{~Hz}$ \\
\hline 12 & Bird & $426.41 \mathrm{~Hz}$ & $1479.08 \mathrm{~Hz}$ \\
\hline 13 & Bite & $654.55 \mathrm{~Hz}$ & $1404.05 \mathrm{~Hz}$ \\
\hline 14 & Bout & $616.60 \mathrm{~Hz}$ & $1316.23 \mathrm{~Hz}$ \\
\hline
\end{tabular}

From table 4.1 above it can be seen that from the 14 words which were pronounced by the native speaker has two ranges $\mathrm{F} 1$ and $\mathrm{F} 2$. A formant is a concentration of acoustic energy around a particular frequency in the speech wave. There are several formants, each at a different frequency, roughly one in each $1000 \mathrm{~Hz}$ band. Or, to put it differently, formants occur at roughly $1000 \mathrm{~Hz}$ intervals. Each formant corresponds to a resonance in the vocal tract. Formants can be seen very clearly in a wideband spectrogram, where they are displayed as dark bands. The darker a formant is reproduced in the spectrogram, the stronger it is (the more energy there is there, or the more audible it is. (Praat.com)

Table 4.2 the tabulation result of the student 1's speech features

\begin{tabular}{|l|l|l|l|l|l|}
\hline No & Word & $\begin{array}{c}\text { First Formant } \\
\text { (F1) }\end{array}$ & $\begin{array}{c}\text { Second Formant } \\
\text { (F2) }\end{array}$ & $\begin{array}{c}\text { Native } \\
\text { speaker's F1 }\end{array}$ & \multicolumn{1}{|c|}{$\begin{array}{c}\text { Native } \\
\text { speaker's F2 }\end{array}$} \\
\hline 1 & Beat & $839.87 \mathrm{~Hz}$ & $2212.47 \mathrm{~Hz}$ & $289.95 \mathrm{~Hz}$ & $2138.92 \mathrm{~Hz}$ \\
\hline 2 & Beard & $698.67 \mathrm{~Hz}$ & $1495.80 \mathrm{~Hz}$ & $348.86 \mathrm{~Hz}$ & $1844.29 \mathrm{~Hz}$ \\
\hline 3 & Bait & $610.63 \mathrm{~Hz}$ & $1613.00 \mathrm{~Hz}$ & $413.11 \mathrm{~Hz}$ & $2023.29 \mathrm{~Hz}$ \\
\hline 4 & Bed & $909.92 \mathrm{~Hz}$ & $1622.32 \mathrm{~Hz}$ & $481.27 \mathrm{~Hz}$ & $1701.05 \mathrm{~Hz}$ \\
\hline 5 & Bad & $961.47 \mathrm{~Hz}$ & $1427.22 \mathrm{~Hz}$ & $654.13 \mathrm{~Hz}$ & $1462.38 \mathrm{~Hz}$ \\
\hline 6 & Body & $830.04 \mathrm{~Hz}$ & $1402.50 \mathrm{~Hz}$ & $537.39 \mathrm{~Hz}$ & $1626.92 \mathrm{~Hz}$ \\
\hline 7 & Bought & $742.98 \mathrm{~Hz}$ & $1311.10 \mathrm{~Hz}$ & $597.49 \mathrm{~Hz}$ & $1126.54 \mathrm{~Hz}$ \\
\hline 8 & Buddhist & $840.20 \mathrm{~Hz}$ & $1797.08 \mathrm{~Hz}$ & $811.43 \mathrm{~Hz}$ & $2114.70 \mathrm{~Hz}$ \\
\hline 9 & Boat & $652.74 \mathrm{~Hz}$ & $1344.75 \mathrm{~Hz}$ & $448.63 \mathrm{~Hz}$ & $1367.80 \mathrm{~Hz}$ \\
\hline 10 & Boot & $624.75 \mathrm{~Hz}$ & $1413.69 \mathrm{~Hz}$ & $352.38 \mathrm{~Hz}$ & $1499.64 \mathrm{~Hz}$ \\
\hline 11 & Bud & $967.55 \mathrm{~Hz}$ & $1377.38 \mathrm{~Hz}$ & $546.17 \mathrm{~Hz}$ & $1453.14 \mathrm{~Hz}$ \\
\hline 12 & Bird & $603.85 \mathrm{~Hz}$ & $1243.82 \mathrm{~Hz}$ & $426.41 \mathrm{~Hz}$ & $1479.08 \mathrm{~Hz}$ \\
\hline 13 & Bite & $832.72 \mathrm{~Hz}$ & $1235.22 \mathrm{~Hz}$ & $654.55 \mathrm{~Hz}$ & $1404.05 \mathrm{~Hz}$ \\
\hline 14 & Bout & $849.49 \mathrm{~Hz}$ & $1188.49 \mathrm{~Hz}$ & $616.60 \mathrm{~Hz}$ & $1316.23 \mathrm{~Hz}$ \\
\hline
\end{tabular}


Table 4.2 shows that the student 1 has higher both F1 and F2 for the 14 words list compared to that of native speaker's speech features. Student 1 tends to have higher position of the vowels sound as presented in F1 column. For example vowel /e/ in Bed,

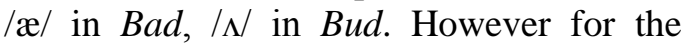

word Buddhist with vowel $/ \mathrm{u} /$ and $/ \mathrm{I} /$ student 1 pronounced relatively close to that of native speaker as it can be seen by the close spectrum scores. While for the Beat with vowel /i/ student 1 has very different range both for F1 and F2 compared to that of native speaker.

Table 4.3 the tabulation result of the student 2's speech features

\begin{tabular}{|l|l|l|l|l|l|}
\hline No & \multicolumn{1}{|c|}{ Word } & $\begin{array}{c}\text { First Formant } \\
\text { (F1) }\end{array}$ & $\begin{array}{c}\text { Second Formant } \\
\text { (F2) }\end{array}$ & $\begin{array}{c}\text { Native } \\
\text { speaker's F1 }\end{array}$ & $\begin{array}{c}\text { Native } \\
\text { speaker's } \\
\text { F2 }\end{array}$ \\
\hline 1 & Beat & $556.04 \mathrm{~Hz}$ & $2100.24 \mathrm{~Hz}$ & $289.95 \mathrm{~Hz}$ & $2138.92 \mathrm{~Hz}$ \\
\hline 2 & Beard & $661.58 \mathrm{~Hz}$ & $1670.00 \mathrm{~Hz}$ & $348.86 \mathrm{~Hz}$ & $1844.29 \mathrm{~Hz}$ \\
\hline 3 & Bait & $850.90 \mathrm{~Hz}$ & $1953.45 \mathrm{~Hz}$ & $413.11 \mathrm{~Hz}$ & $2023.29 \mathrm{~Hz}$ \\
\hline 4 & Bed & $790.25 \mathrm{~Hz}$ & $1473.59 \mathrm{~Hz}$ & $481.27 \mathrm{~Hz}$ & $1701.05 \mathrm{~Hz}$ \\
\hline 5 & Bad & $829.66 \mathrm{~Hz}$ & $1452.48 \mathrm{~Hz}$ & $654.13 \mathrm{~Hz}$ & $1462.38 \mathrm{~Hz}$ \\
\hline 6 & Body & $701.83 \mathrm{~Hz}$ & $1207.94 \mathrm{~Hz}$ & $537.39 \mathrm{~Hz}$ & $1626.92 \mathrm{~Hz}$ \\
\hline 7 & Bought & $535.13 \mathrm{~Hz}$ & $1147.29 \mathrm{~Hz}$ & $597.49 \mathrm{~Hz}$ & $1126.54 \mathrm{~Hz}$ \\
\hline 8 & Buddhist & $823.06 \mathrm{~Hz}$ & $1677.88 \mathrm{~Hz}$ & $811.43 \mathrm{~Hz}$ & $2114.70 \mathrm{~Hz}$ \\
\hline 9 & Boat & $550.74 \mathrm{~Hz}$ & $1193.50 \mathrm{~Hz}$ & $448.63 \mathrm{~Hz}$ & $1367.80 \mathrm{~Hz}$ \\
\hline 10 & Boot & $508.19 \mathrm{~Hz}$ & $1162.28 \mathrm{~Hz}$ & $352.38 \mathrm{~Hz}$ & $1499.64 \mathrm{~Hz}$ \\
\hline 11 & Bud & $824.84 \mathrm{~Hz}$ & $1290.58 \mathrm{~Hz}$ & $546.17 \mathrm{~Hz}$ & $1453.14 \mathrm{~Hz}$ \\
\hline 12 & Bird & $728.93 \mathrm{~Hz}$ & $1408.17 \mathrm{~Hz}$ & $426.41 \mathrm{~Hz}$ & $1479.08 \mathrm{~Hz}$ \\
\hline 13 & Bite & $761.23 \mathrm{~Hz}$ & $1397.04 \mathrm{~Hz}$ & $654.55 \mathrm{~Hz}$ & $1404.05 \mathrm{~Hz}$ \\
\hline 14 & Bout & $750.09 \mathrm{~Hz}$ & $1139.34 \mathrm{~Hz}$ & $616.60 \mathrm{~Hz}$ & $1316.23 \mathrm{~Hz}$ \\
\hline
\end{tabular}

From 4.3 student 2 also tends to have higher F1 and F2 compared to that of native speaker. However he has four words which are close in range to that of native speaker: Bought /o/, Buddhist /\%//I/, Boat /o/, Bite $/ a \mathrm{I} /$.

Table 4.4 the tabulation result of the student 3's speech features

\begin{tabular}{|l|l|l|l|l|l|}
\hline No & \multicolumn{1}{|c|}{ Word } & $\begin{array}{c}\text { First Formant } \\
\text { (F1) }\end{array}$ & $\begin{array}{c}\text { Second Formant } \\
\text { (F2) }\end{array}$ & $\begin{array}{c}\text { Native } \\
\text { speaker's } \\
\text { F1 }\end{array}$ & $\begin{array}{c}\text { Native } \\
\text { speaker's } \\
\text { F2 }\end{array}$ \\
\hline 1 & Beat & $610.39 \mathrm{~Hz}$ & $1961.07 \mathrm{~Hz}$ & $289.95 \mathrm{~Hz}$ & $2138.92 \mathrm{~Hz}$ \\
\hline 2 & Beard & $578.65 \mathrm{~Hz}$ & $1912.05 \mathrm{~Hz}$ & $348.86 \mathrm{~Hz}$ & $1844.29 \mathrm{~Hz}$ \\
\hline 3 & Bait & $576.80 \mathrm{~Hz}$ & $1410.38 \mathrm{~Hz}$ & $413.11 \mathrm{~Hz}$ & $2023.29 \mathrm{~Hz}$ \\
\hline 4 & Bed & $858.14 \mathrm{~Hz}$ & $1430.65 \mathrm{~Hz}$ & $481.27 \mathrm{~Hz}$ & $1701.05 \mathrm{~Hz}$ \\
\hline 5 & Bad & $816.83 \mathrm{~Hz}$ & $1147.77 \mathrm{~Hz}$ & $654.13 \mathrm{~Hz}$ & $1462.38 \mathrm{~Hz}$ \\
\hline 6 & Body & $714.78 \mathrm{~Hz}$ & $1306.92 \mathrm{~Hz}$ & $537.39 \mathrm{~Hz}$ & $1626.92 \mathrm{~Hz}$ \\
\hline 7 & Bought & $720.07 \mathrm{~Hz}$ & $1173.38 \mathrm{~Hz}$ & $597.49 \mathrm{~Hz}$ & $1126.54 \mathrm{~Hz}$ \\
\hline 8 & Buddhist & $782.36 \mathrm{~Hz}$ & $1796.30 \mathrm{~Hz}$ & $811.43 \mathrm{~Hz}$ & $2114.70 \mathrm{~Hz}$ \\
\hline 9 & Boat & $622.83 \mathrm{~Hz}$ & $1160.77 \mathrm{~Hz}$ & $448.63 \mathrm{~Hz}$ & $1367.80 \mathrm{~Hz}$ \\
\hline 10 & Boot & $570.08 \mathrm{~Hz}$ & $1170.54 \mathrm{~Hz}$ & $352.38 \mathrm{~Hz}$ & $1499.64 \mathrm{~Hz}$ \\
\hline 11 & Bud & $951.19 \mathrm{~Hz}$ & $1347.69 \mathrm{~Hz}$ & $546.17 \mathrm{~Hz}$ & $1453.14 \mathrm{~Hz}$ \\
\hline 12 & Bird & $673.33 \mathrm{~Hz}$ & $1313.07 \mathrm{~Hz}$ & $426.41 \mathrm{~Hz}$ & $1479.08 \mathrm{~Hz}$ \\
\hline 13 & Bite & $870.45 \mathrm{~Hz}$ & $1214.88 \mathrm{~Hz}$ & $654.55 \mathrm{~Hz}$ & $1404.05 \mathrm{~Hz}$ \\
\hline 14 & Bout & $708.44 \mathrm{~Hz}$ & $1170.60 \mathrm{~Hz}$ & $616.60 \mathrm{~Hz}$ & $1316.23 \mathrm{~Hz}$ \\
\hline
\end{tabular}


From table 4.4 it can be seen student 3 also has higher F1 and F2 compare to that of native speaker. The interesting points that can be taken from this tabulation, for vowel /i/ in Beat, /e/ in Bed, / $/ \mathrm{A}$ in Bud she has very high F1. This due to in accuracy in pronouncing those vowel sounds. However, she has quite similar in pronouncing vowel $/ \mho /$ and $/ \mathrm{I} /$ in Buddhist compared to that of native $/ \mathrm{u} /$ and $/ \mathrm{I} /$.

Table 4.5 the tabulation result of the student 4's speech features

\begin{tabular}{|l|l|l|l|l|l|}
\hline No & \multicolumn{1}{|c|}{ Word } & $\begin{array}{c}\text { First Formant } \\
\text { (F1) }\end{array}$ & $\begin{array}{c}\text { Second Formant } \\
\text { (F2) }\end{array}$ & $\begin{array}{c}\text { Native } \\
\text { speaker's } \\
\text { F1 }\end{array}$ & $\begin{array}{c}\text { Native } \\
\text { speaker's } \\
\text { F2 }\end{array}$ \\
\hline 1 & Beat & $568.56 \mathrm{~Hz}$ & $2450.96 \mathrm{~Hz}$ & $289.95 \mathrm{~Hz}$ & $2138.92 \mathrm{~Hz}$ \\
\hline 2 & Beard & $521.48 \mathrm{~Hz}$ & $1648.14 \mathrm{~Hz}$ & $348.86 \mathrm{~Hz}$ & $1844.29 \mathrm{~Hz}$ \\
\hline 3 & Bait & $518.60 \mathrm{~Hz}$ & $2326.37 \mathrm{~Hz}$ & $413.11 \mathrm{~Hz}$ & $2023.29 \mathrm{~Hz}$ \\
\hline 4 & Bed & $728.68 \mathrm{~Hz}$ & $1761.64 \mathrm{~Hz}$ & $481.27 \mathrm{~Hz}$ & $1701.05 \mathrm{~Hz}$ \\
\hline 5 & Bad & $683.10 \mathrm{~Hz}$ & $1828.27 \mathrm{~Hz}$ & $654.13 \mathrm{~Hz}$ & $1462.38 \mathrm{~Hz}$ \\
\hline 6 & Body & $702.78 \mathrm{~Hz}$ & $1817.53 \mathrm{~Hz}$ & $537.39 \mathrm{~Hz}$ & $1626.92 \mathrm{~Hz}$ \\
\hline 7 & Bought & $673.17 \mathrm{~Hz}$ & $1165.54 \mathrm{~Hz}$ & $597.49 \mathrm{~Hz}$ & $1126.54 \mathrm{~Hz}$ \\
\hline 8 & Buddhist & $737.08 \mathrm{~Hz}$ & $2131.26 \mathrm{~Hz}$ & $811.43 \mathrm{~Hz}$ & $2114.70 \mathrm{~Hz}$ \\
\hline 9 & Boat & $513.41 \mathrm{~Hz}$ & $1084.35 \mathrm{~Hz}$ & $448.63 \mathrm{~Hz}$ & $1367.80 \mathrm{~Hz}$ \\
\hline 10 & Boot & $500.77 \mathrm{~Hz}$ & $1125.97 \mathrm{~Hz}$ & $352.38 \mathrm{~Hz}$ & $1499.64 \mathrm{~Hz}$ \\
\hline 11 & Bud & $785.28 \mathrm{~Hz}$ & $1426.32 \mathrm{~Hz}$ & $546.17 \mathrm{~Hz}$ & $1453.14 \mathrm{~Hz}$ \\
\hline 12 & Bird & $583.41 \mathrm{~Hz}$ & $1384.14 \mathrm{~Hz}$ & $426.41 \mathrm{~Hz}$ & $1479.08 \mathrm{~Hz}$ \\
\hline 13 & Bite & $764.19 \mathrm{~Hz}$ & $1458.38 \mathrm{~Hz}$ & $654.55 \mathrm{~Hz}$ & $1404.05 \mathrm{~Hz}$ \\
\hline 14 & Bout & $605.62 \mathrm{~Hz}$ & $1130.75 \mathrm{~Hz}$ & $616.60 \mathrm{~Hz}$ & $1316.23 \mathrm{~Hz}$ \\
\hline
\end{tabular}

From Table 4.5 it can be seen that student 4 has relatively close score range for all 14 words compare to that of native speaker speech feature. However she has quite higher formant scores for vowel sounds /i/ in Beat, /e/ in Bed, /o/ in Body, / $/ /$ in Bud. Interestingly, here the $/ \mho /$ and $/ \mathrm{I} /$ vowel sounds in Buddhist are in very close range score to that of native speaker.

Table 4.6 the tabulation result of the student 5's speech features

\begin{tabular}{|l|l|l|l|l|l|}
\hline No & \multicolumn{1}{|c|}{ Word } & $\begin{array}{c}\text { First Formant } \\
\text { (F1) }\end{array}$ & $\begin{array}{c}\text { Second Formant } \\
\text { (F2) }\end{array}$ & $\begin{array}{c}\text { Native } \\
\text { speaker's } \\
\text { F1 }\end{array}$ & $\begin{array}{c}\text { Native } \\
\text { speaker's } \\
\text { F2 }\end{array}$ \\
\hline 1 & Beat & $402.66 \mathrm{~Hz}$ & $1191.81 \mathrm{~Hz}$ & $289.95 \mathrm{~Hz}$ & $2138.92 \mathrm{~Hz}$ \\
\hline 2 & Beard & $572.45 \mathrm{~Hz}$ & $1930.45 \mathrm{~Hz}$ & $348.86 \mathrm{~Hz}$ & $1844.29 \mathrm{~Hz}$ \\
\hline 3 & Bait & $576.93 \mathrm{~Hz}$ & $1233.09 \mathrm{~Hz}$ & $413.11 \mathrm{~Hz}$ & $2023.29 \mathrm{~Hz}$ \\
\hline 4 & Bed & $936.88 \mathrm{~Hz}$ & $1226.87 \mathrm{~Hz}$ & $481.27 \mathrm{~Hz}$ & $1701.05 \mathrm{~Hz}$ \\
\hline 5 & Bad & $885.83 \mathrm{~Hz}$ & $1183.18 \mathrm{~Hz}$ & $654.13 \mathrm{~Hz}$ & $1462.38 \mathrm{~Hz}$ \\
\hline 6 & Body & $718.64 \mathrm{~Hz}$ & $1362.14 \mathrm{~Hz}$ & $537.39 \mathrm{~Hz}$ & $1626.92 \mathrm{~Hz}$ \\
\hline 7 & Bought & $714.77 \mathrm{~Hz}$ & $1275.17 \mathrm{~Hz}$ & $597.49 \mathrm{~Hz}$ & $1126.54 \mathrm{~Hz}$ \\
\hline 8 & Buddhist & $875.96 \mathrm{~Hz}$ & $1799.62 \mathrm{~Hz}$ & $811.43 \mathrm{~Hz}$ & $2114.70 \mathrm{~Hz}$ \\
\hline 9 & Boat & $641.61 \mathrm{~Hz}$ & $1148.20 \mathrm{~Hz}$ & $448.63 \mathrm{~Hz}$ & $1367.80 \mathrm{~Hz}$ \\
\hline 10 & Boot & $628.56 \mathrm{~Hz}$ & $1360.93 \mathrm{~Hz}$ & $352.38 \mathrm{~Hz}$ & $1499.64 \mathrm{~Hz}$ \\
\hline 11 & Bud & $835.94 \mathrm{~Hz}$ & $1282.78 \mathrm{~Hz}$ & $546.17 \mathrm{~Hz}$ & $1453.14 \mathrm{~Hz}$ \\
\hline 12 & Bird & $601.39 \mathrm{~Hz}$ & $1171.81 \mathrm{~Hz}$ & $426.41 \mathrm{~Hz}$ & $1479.08 \mathrm{~Hz}$ \\
\hline 13 & Bite & $814.45 \mathrm{~Hz}$ & $1251.31 \mathrm{~Hz}$ & $654.55 \mathrm{~Hz}$ & $1404.05 \mathrm{~Hz}$ \\
\hline 14 & Bout & $906.32 \mathrm{~Hz}$ & $1292.50 \mathrm{~Hz}$ & $616.60 \mathrm{~Hz}$ & $1316.23 \mathrm{~Hz}$ \\
\hline
\end{tabular}


From Table 4.6 it obvious that for certain vowel sounds feature, student 6 has higher F1 and F2 compared to that of native speaker. For example the vowel /e/ in Bed she tended to be away higher for the F1 with lower F2. The same pattern was also found for vowel $/ \Lambda /$ in Bud and vowel /ov/ in Bout. Somehow student 6 could pronounce almost equal in spectrum range to that of native speaker for vowel sounds $/ \mathrm{v} /$ and $/ \mathrm{I} /$ in Buddhist.

Table 4.7 the tabulation result of the student 6's speech features

\begin{tabular}{|l|l|l|l|l|l|}
\hline No & \multicolumn{1}{|c|}{ Word } & $\begin{array}{c}\text { First Formant } \\
\text { (F1) }\end{array}$ & $\begin{array}{c}\text { Second Formant } \\
\text { (F2) }\end{array}$ & $\begin{array}{c}\text { Native } \\
\text { speaker's } \\
\text { F1 }\end{array}$ & $\begin{array}{c}\text { Native } \\
\text { speaker's } \\
\text { F2 }\end{array}$ \\
\hline 1 & Beat & $426.48 \mathrm{~Hz}$ & $1877.61 \mathrm{~Hz}$ & $289.95 \mathrm{~Hz}$ & $2138.92 \mathrm{~Hz}$ \\
\hline 2 & Beard & $725.03 \mathrm{~Hz}$ & $1688.77 \mathrm{~Hz}$ & $348.86 \mathrm{~Hz}$ & $1844.29 \mathrm{~Hz}$ \\
\hline 3 & Bait & $466.21 \mathrm{~Hz}$ & $1958.61 \mathrm{~Hz}$ & $413.11 \mathrm{~Hz}$ & $2023.29 \mathrm{~Hz}$ \\
\hline 4 & Bed & $741.64 \mathrm{~Hz}$ & $1709.71 \mathrm{~Hz}$ & $481.27 \mathrm{~Hz}$ & $1701.05 \mathrm{~Hz}$ \\
\hline 5 & Bad & $721.99 \mathrm{~Hz}$ & $1990.65 \mathrm{~Hz}$ & $654.13 \mathrm{~Hz}$ & $1462.38 \mathrm{~Hz}$ \\
\hline 6 & Body & $916.14 \mathrm{~Hz}$ & $2372.12 \mathrm{~Hz}$ & $537.39 \mathrm{~Hz}$ & $1626.92 \mathrm{~Hz}$ \\
\hline 7 & Bought & $379.84 \mathrm{~Hz}$ & $1047.38 \mathrm{~Hz}$ & $597.49 \mathrm{~Hz}$ & $1126.54 \mathrm{~Hz}$ \\
\hline 8 & Buddhist & $744.19 \mathrm{~Hz}$ & $2176.38 \mathrm{~Hz}$ & $811.43 \mathrm{~Hz}$ & $2114.70 \mathrm{~Hz}$ \\
\hline 9 & Boat & $535.33 \mathrm{~Hz}$ & $1266.36 \mathrm{~Hz}$ & $448.63 \mathrm{~Hz}$ & $1367.80 \mathrm{~Hz}$ \\
\hline 10 & Boot & $389.56 \mathrm{~Hz}$ & $1176.85 \mathrm{~Hz}$ & $352.38 \mathrm{~Hz}$ & $1499.64 \mathrm{~Hz}$ \\
\hline 11 & Bud & $874.93 \mathrm{~Hz}$ & $1439.91 \mathrm{~Hz}$ & $546.17 \mathrm{~Hz}$ & $1453.14 \mathrm{~Hz}$ \\
\hline 12 & Bird & $439.05 \mathrm{~Hz}$ & $1400.70 \mathrm{~Hz}$ & $426.41 \mathrm{~Hz}$ & $1479.08 \mathrm{~Hz}$ \\
\hline 13 & Bite & $760.22 \mathrm{~Hz}$ & $1386.76 \mathrm{~Hz}$ & $654.55 \mathrm{~Hz}$ & $1404.05 \mathrm{~Hz}$ \\
\hline 14 & Bout & $757.71 \mathrm{~Hz}$ & $1226.95 \mathrm{~Hz}$ & $616.60 \mathrm{~Hz}$ & $1316.23 \mathrm{~Hz}$ \\
\hline
\end{tabular}

From table 4.7 some point can be commented on how student 6 spelling the vowel sounds for the words list. Inaccuracy of some vowel sounds can be seen from the higher F1 and F2 score range compared to that of native speaker. The vowel /ı/ in Beard, / $/$ / in Body and $/ N$ in Bud are the examples. There is one vowel segment which is lower both F1 and F2 / o / in Bought. Despite of those inaccuracies, she could maintain very close spectrum ranges to native speaker for some other vowel sounds. They are: /es/ in Bait, /o/ in Boat, /u/ in Boot, /a/ in Bird.

Table 4.8 the tabulation result of the student 7's speech features

\begin{tabular}{|l|l|l|l|l|l|}
\hline No & \multicolumn{1}{|c|}{ Word } & $\begin{array}{c}\text { First Formant } \\
\text { (F1) }\end{array}$ & $\begin{array}{c}\text { Second Formant } \\
\text { (F2) }\end{array}$ & $\begin{array}{c}\text { Native } \\
\text { speaker's } \\
\text { F1 }\end{array}$ & $\begin{array}{c}\text { Native } \\
\text { speaker's } \\
\text { F2 }\end{array}$ \\
\hline 1 & Beat & $596.26 \mathrm{~Hz}$ & $1698.54 \mathrm{~Hz}$ & $289.95 \mathrm{~Hz}$ & $2138.92 \mathrm{~Hz}$ \\
\hline 2 & Beard & $754.02 \mathrm{~Hz}$ & $1808.63 \mathrm{~Hz}$ & $348.86 \mathrm{~Hz}$ & $1844.29 \mathrm{~Hz}$ \\
\hline 3 & Bait & $719.53 \mathrm{~Hz}$ & $1600.40 \mathrm{~Hz}$ & $413.11 \mathrm{~Hz}$ & $2023.29 \mathrm{~Hz}$ \\
\hline 4 & Bed & $909.57 \mathrm{~Hz}$ & $1358.47 \mathrm{~Hz}$ & $481.27 \mathrm{~Hz}$ & $1701.05 \mathrm{~Hz}$ \\
\hline 5 & Bad & $964.31 \mathrm{~Hz}$ & $1412.01 \mathrm{~Hz}$ & $654.13 \mathrm{~Hz}$ & $1462.38 \mathrm{~Hz}$ \\
\hline 6 & Body & $700.12 \mathrm{~Hz}$ & $1307.86 \mathrm{~Hz}$ & $537.39 \mathrm{~Hz}$ & $1626.92 \mathrm{~Hz}$ \\
\hline 7 & Bought & $798.69 \mathrm{~Hz}$ & $1384.35 \mathrm{~Hz}$ & $597.49 \mathrm{~Hz}$ & $1126.54 \mathrm{~Hz}$ \\
\hline 8 & Buddhist & $862.54 \mathrm{~Hz}$ & $2078.55 \mathrm{~Hz}$ & $811.43 \mathrm{~Hz}$ & $2114.70 \mathrm{~Hz}$ \\
\hline 9 & Boat & $768.10 \mathrm{~Hz}$ & $1474.98 \mathrm{~Hz}$ & $448.63 \mathrm{~Hz}$ & $1367.80 \mathrm{~Hz}$ \\
\hline 10 & Boot & $618.34 \mathrm{~Hz}$ & $1308.00 \mathrm{~Hz}$ & $352.38 \mathrm{~Hz}$ & $1499.64 \mathrm{~Hz}$ \\
\hline 11 & Bud & $906.59 \mathrm{~Hz}$ & $1449.34 \mathrm{~Hz}$ & $546.17 \mathrm{~Hz}$ & $1453.14 \mathrm{~Hz}$ \\
\hline 12 & Bird & $606.64 \mathrm{~Hz}$ & $1348.81 \mathrm{~Hz}$ & $426.41 \mathrm{~Hz}$ & $1479.08 \mathrm{~Hz}$ \\
\hline 13 & Bite & $909.02 \mathrm{~Hz}$ & $1619.72 \mathrm{~Hz}$ & $654.55 \mathrm{~Hz}$ & $1404.05 \mathrm{~Hz}$ \\
\hline 14 & Bout & $929.65 \mathrm{~Hz}$ & $1603.70 \mathrm{~Hz}$ & $616.60 \mathrm{~Hz}$ & $1316.23 \mathrm{~Hz}$ \\
\hline
\end{tabular}


From the table 4.8 it can be seen that student 7 also has a tendency to have F1 and F2 higher from that of native speaker. Even to some vowel sounds are so high in the range of the spectrum compared to the spectrum of the native speaker's F1 and F2. Those inaccuracies can be seen in the following vowel sounds: /e/ in Bed, /æ/ in
Bad, / $/$ in Bud, /ai/ in Bite, /ov/ Bout. However there is one word out of the 14 words list, which student 7 could manage to the acceptable spectrum range and closely to the spectrum produced by the native speaker, which are vowel $/ \mho /$ and $/ \mathrm{I} /$ in Buddhist.

Table 4.9 the tabulation result of the student 8's speech features

\begin{tabular}{|l|l|l|l|l|l|}
\hline No & \multicolumn{1}{|c|}{ Word } & $\begin{array}{c}\text { First Formant } \\
\text { (F1) }\end{array}$ & $\begin{array}{c}\text { Second Formant } \\
\text { (F2) }\end{array}$ & $\begin{array}{c}\text { Native } \\
\text { speaker's } \\
\text { F1 }\end{array}$ & $\begin{array}{c}\text { Native } \\
\text { speaker's } \\
\text { F2 }\end{array}$ \\
\hline 1 & Beat & $436.49 \mathrm{~Hz}$ & $1890.14 \mathrm{~Hz}$ & $289.95 \mathrm{~Hz}$ & $2138.92 \mathrm{~Hz}$ \\
\hline 2 & Beard & $533.41 \mathrm{~Hz}$ & $1490.08 \mathrm{~Hz}$ & $348.86 \mathrm{~Hz}$ & $1844.29 \mathrm{~Hz}$ \\
\hline 3 & Bait & $565.18 \mathrm{~Hz}$ & $1681.78 \mathrm{~Hz}$ & $413.11 \mathrm{~Hz}$ & $2023.29 \mathrm{~Hz}$ \\
\hline 4 & Bed & $694.03 \mathrm{~Hz}$ & $1352.92 \mathrm{~Hz}$ & $481.27 \mathrm{~Hz}$ & $1701.05 \mathrm{~Hz}$ \\
\hline 5 & Bad & $719.51 \mathrm{~Hz}$ & $1305.03 \mathrm{~Hz}$ & $654.13 \mathrm{~Hz}$ & $1462.38 \mathrm{~Hz}$ \\
\hline 6 & Body & $690.44 \mathrm{~Hz}$ & $1299.32 \mathrm{~Hz}$ & $537.39 \mathrm{~Hz}$ & $1626.92 \mathrm{~Hz}$ \\
\hline 7 & Bought & $502.88 \mathrm{~Hz}$ & $1106.02 \mathrm{~Hz}$ & $597.49 \mathrm{~Hz}$ & $1126.54 \mathrm{~Hz}$ \\
\hline 8 & Buddhist & $675.93 \mathrm{~Hz}$ & $1980.56 \mathrm{~Hz}$ & $811.43 \mathrm{~Hz}$ & $2114.70 \mathrm{~Hz}$ \\
\hline 9 & Boat & $502.06 \mathrm{~Hz}$ & $1183.39 \mathrm{~Hz}$ & $448.63 \mathrm{~Hz}$ & $1367.80 \mathrm{~Hz}$ \\
\hline 10 & Boot & $403.45 \mathrm{~Hz}$ & $1226.02 \mathrm{~Hz}$ & $352.38 \mathrm{~Hz}$ & $1499.64 \mathrm{~Hz}$ \\
\hline 11 & Bud & $658.87 \mathrm{~Hz}$ & $1227.38 \mathrm{~Hz}$ & $546.17 \mathrm{~Hz}$ & $1453.14 \mathrm{~Hz}$ \\
\hline 12 & Bird & $497.09 \mathrm{~Hz}$ & $1268.16 \mathrm{~Hz}$ & $426.41 \mathrm{~Hz}$ & $1479.08 \mathrm{~Hz}$ \\
\hline 13 & Bite & $745.74 \mathrm{~Hz}$ & $1241.69 \mathrm{~Hz}$ & $654.55 \mathrm{~Hz}$ & $1404.05 \mathrm{~Hz}$ \\
\hline 14 & Bout & $519.92 \mathrm{~Hz}$ & $1113.82 \mathrm{~Hz}$ & $616.60 \mathrm{~Hz}$ & $1316.23 \mathrm{~Hz}$ \\
\hline
\end{tabular}

From table 4.9 it can be seen that student 8 relatively has close spectrum range to that of native speaker. Despite of that, some inaccuracy are found whether in higher and lower degree of spectrum F1 and F2 compared to that of native speaker. For example vowel /æ/ in $\mathrm{Bad}$ is higher, while $/ \mathrm{o} /$ and $/ \mathrm{I} /$ in Buddhist, /ai/ in Bite, and / $\mathrm{o} /$ in Bout are lower in range.

Table 4.10 the tabulation result of the student 9's speech features

\begin{tabular}{|l|l|l|l|l|l|}
\hline No & \multicolumn{1}{|c|}{$\begin{array}{c}\text { Word } \\
\text { First Formant }\end{array}$} & $\begin{array}{c}\text { Second Formant } \\
\text { (F2) }\end{array}$ & $\begin{array}{c}\text { Native } \\
\text { speaker's } \\
\text { F1 }\end{array}$ & $\begin{array}{c}\text { Native } \\
\text { speaker's } \\
\text { F2 }\end{array}$ \\
\hline 1 & Beat & $435.90 \mathrm{~Hz}$ & $1847.27 \mathrm{~Hz}$ & $289.95 \mathrm{~Hz}$ & $2138.92 \mathrm{~Hz}$ \\
\hline 2 & Beard & $654.38 \mathrm{~Hz}$ & $1404.54 \mathrm{~Hz}$ & $348.86 \mathrm{~Hz}$ & $1844.29 \mathrm{~Hz}$ \\
\hline 3 & Bait & $407.61 \mathrm{~Hz}$ & $1926.24 \mathrm{~Hz}$ & $413.11 \mathrm{~Hz}$ & $2023.29 \mathrm{~Hz}$ \\
\hline 4 & Bed & $653.86 \mathrm{~Hz}$ & $1626.89 \mathrm{~Hz}$ & $481.27 \mathrm{~Hz}$ & $1701.05 \mathrm{~Hz}$ \\
\hline 5 & Bad & $672.16 \mathrm{~Hz}$ & $1717.69 \mathrm{~Hz}$ & $654.13 \mathrm{~Hz}$ & $1462.38 \mathrm{~Hz}$ \\
\hline 6 & Body & $645.07 \mathrm{~Hz}$ & $1358.14 \mathrm{~Hz}$ & $537.39 \mathrm{~Hz}$ & $1626.92 \mathrm{~Hz}$ \\
\hline 7 & Bought & $469.23 \mathrm{~Hz}$ & $1032.92 \mathrm{~Hz}$ & $597.49 \mathrm{~Hz}$ & $1126.54 \mathrm{~Hz}$ \\
\hline 8 & Buddhist & $820.78 \mathrm{~Hz}$ & $2213.12 \mathrm{~Hz}$ & $811.43 \mathrm{~Hz}$ & $2114.70 \mathrm{~Hz}$ \\
\hline 9 & Boat & $429.96 \mathrm{~Hz}$ & $1009.09 \mathrm{~Hz}$ & $448.63 \mathrm{~Hz}$ & $1367.80 \mathrm{~Hz}$ \\
\hline 10 & Boot & $541.33 \mathrm{~Hz}$ & $1224.36 \mathrm{~Hz}$ & $352.38 \mathrm{~Hz}$ & $1499.64 \mathrm{~Hz}$ \\
\hline 11 & Bud & $638.78 \mathrm{~Hz}$ & $1292.25 \mathrm{~Hz}$ & $546.17 \mathrm{~Hz}$ & $1453.14 \mathrm{~Hz}$ \\
\hline 12 & Bird & $836.41 \mathrm{~Hz}$ & $1417.75 \mathrm{~Hz}$ & $426.41 \mathrm{~Hz}$ & $1479.08 \mathrm{~Hz}$ \\
\hline 13 & Bite & $760.89 \mathrm{~Hz}$ & $1229.94 \mathrm{~Hz}$ & $654.55 \mathrm{~Hz}$ & $1404.05 \mathrm{~Hz}$ \\
\hline 14 & Bout & $502.14 \mathrm{~Hz}$ & $1013.57 \mathrm{~Hz}$ & $616.60 \mathrm{~Hz}$ & $1316.23 \mathrm{~Hz}$ \\
\hline
\end{tabular}


From table 4.10 it can be seen that student 9 also relatively has very close spectrum range to that of native speaker. Only one word that is higher in the F1 for vowel /ə/ in. While / / / and /// in Buddhist stay within the same spectrum range.

Table 4.11 the tabulation result of the student 10's speech features

\begin{tabular}{|l|l|l|l|l|l|}
\hline No & \multicolumn{1}{|c|}{ Word } & $\begin{array}{c}\text { First Formant } \\
\text { (F1) }\end{array}$ & $\begin{array}{c}\text { Second Formant } \\
\text { (F2) }\end{array}$ & $\begin{array}{c}\text { Native } \\
\text { speaker's } \\
\text { F1 }\end{array}$ & $\begin{array}{c}\text { Native } \\
\text { speaker's F2 }\end{array}$ \\
\hline 1 & Beat & $729.78 \mathrm{~Hz}$ & $2400.37 \mathrm{~Hz}$ & $289.95 \mathrm{~Hz}$ & $2138.92 \mathrm{~Hz}$ \\
\hline 2 & Beard & $782.75 \mathrm{~Hz}$ & $2074.24 \mathrm{~Hz}$ & $348.86 \mathrm{~Hz}$ & $1844.29 \mathrm{~Hz}$ \\
\hline 3 & Bait & $634.85 \mathrm{~Hz}$ & $1753.06 \mathrm{~Hz}$ & $413.11 \mathrm{~Hz}$ & $2023.29 \mathrm{~Hz}$ \\
\hline 4 & Bed & $882.87 \mathrm{~Hz}$ & $1352.96 \mathrm{~Hz}$ & $481.27 \mathrm{~Hz}$ & $1701.05 \mathrm{~Hz}$ \\
\hline 5 & Bad & $928.52 \mathrm{~Hz}$ & $1352.20 \mathrm{~Hz}$ & $654.13 \mathrm{~Hz}$ & $1462.38 \mathrm{~Hz}$ \\
\hline 6 & Body & $625.19 \mathrm{~Hz}$ & $1677.02 \mathrm{~Hz}$ & $537.39 \mathrm{~Hz}$ & $1626.92 \mathrm{~Hz}$ \\
\hline 7 & Bought & $528.35 \mathrm{~Hz}$ & $1112.47 \mathrm{~Hz}$ & $597.49 \mathrm{~Hz}$ & $1126.54 \mathrm{~Hz}$ \\
\hline 8 & Buddhist & $710.77 \mathrm{~Hz}$ & $1928.63 \mathrm{~Hz}$ & $811.43 \mathrm{~Hz}$ & $2114.70 \mathrm{~Hz}$ \\
\hline 9 & Boat & $763.02 \mathrm{~Hz}$ & $1487.08 \mathrm{~Hz}$ & $448.63 \mathrm{~Hz}$ & $1367.80 \mathrm{~Hz}$ \\
\hline 10 & Boot & $419.47 \mathrm{~Hz}$ & $1113.25 \mathrm{~Hz}$ & $352.38 \mathrm{~Hz}$ & $1499.64 \mathrm{~Hz}$ \\
\hline 11 & Bud & $907.82 \mathrm{~Hz}$ & $1299.00 \mathrm{~Hz}$ & $546.17 \mathrm{~Hz}$ & $1453.14 \mathrm{~Hz}$ \\
\hline 12 & Bird & $689.76 \mathrm{~Hz}$ & $1290.68 \mathrm{~Hz}$ & $426.41 \mathrm{~Hz}$ & $1479.08 \mathrm{~Hz}$ \\
\hline 13 & Bite & $810.28 \mathrm{~Hz}$ & $1321.56 \mathrm{~Hz}$ & $654.55 \mathrm{~Hz}$ & $1404.05 \mathrm{~Hz}$ \\
\hline 14 & Bout & $862.88 \mathrm{~Hz}$ & $1257.24 \mathrm{~Hz}$ & $616.60 \mathrm{~Hz}$ & $1316.23 \mathrm{~Hz}$ \\
\hline
\end{tabular}

From table 4.10 it can be seen that student 10 has tendency to have higher F1 and F2, some are even very high compared to that of native speaker. For example vowel /i/ in Beat, /ra/ in Beard, /e/ in Bed, /æ/ in Bad and $/ \Lambda /$ in $B u d$. This is due to inaccuracy in pronunciation.

The overall analysis from table 4.1 to 4.11 indicated that sample of the participant have a tendency to have higher $\mathrm{F} 1$ and $\mathrm{F} 2$ spectrum range scores compared to that of native speaker. In analysing segmental feature of a learner the application of PRAAT software provides method in which voice spectrum can be measured. All vowels can be can be characterized by the first formant (F1) and the second formant (F2). F1 presents the pronunciation accuracy of the high and the low location of the vowel. While the F2 indicates the accuracy of the front and back location of the vowels. Vowels traditionally known as front have F1 and F2 a good distance apart. Vowels traditionally know as back have F1 and F2 so close that they touch. The F1 has a higher frequency for an open vowel (such as [a]) and a lower frequency for a close vowel (such as [i] or $[\mathrm{u}]$ ); and the $\mathrm{F} 2$ has a higher frequency for a front vowel (such as [i]) and a lower frequency for a back vowel (such as [u]. Participants tend to have higher F1 compared to that of native speaker indicates that the higher the formant frequency they produced, the lower the vowel height they reached. This condition is possible because the F1 in vowels is inversely related to vowel height. Similar phenomenon is also happen to the range of F2. The Participants, they tend to "drag" the vowel sound to frontal sounds thus as the consequent they produced higher F2. Since the F2 in vowels is somewhat related to degree of backness, i.e. the more front the vowel, the higher the second formant will be. The different score of F1 and F2 had shown the different level of the vowel stress of the minimal pair's word list. Thus to disambiguate the vowel sounds can be attributed to the differences in their first formant and second formant.

It is also found from the finding that some of the participants have difficulty in distinguishing vowel /e/ in Bed to /æ/ in $\mathrm{Bad}$. Consequently mispronouncing the word is unavoidable. Correspond to the former case, some other participant also 
having a problem in distinguishing vowel $/ \mathrm{u} /$ in Boot to vowel $/ \mathrm{N}$ in Bud. The possible reason affecting these phenomena is the influence of Indonesian vowel system in spelling and pronunciation is different from that of English language. Interestingly almost all of the participant have the spectrum F1 and F2 within the same score range compared to that of native speaker for vowel sounds / $/ \mathrm{J}$ and $/ / /$ in Buddhist. This finding was found might be the word Buddhist /budıst/ is not an original English word and the vowel sounds in the word is pronounced as in Indonesian vowel $/ \mathrm{u} /$ and /i/.

\subsection{Modifiying learners' problematic features.}

After analysing particular problematic segments of students in their pronunciation. Some teaching techniques are suggested to modify their segmental problem.

\section{Teaching segmental:}

To teach segmental features is suggested by Joaquin (2009). She claims that drilling is related to the works of mirror neurons system involved in acquiring speech perception. Moreover, she adds that drilling segmental features as an important aspect of communicative competence in order to avoid miscommunication, embarrassment, which can affect confidence and motivation from the ES/EF learner. Drilling may not be fun for teaching and learning but it is considered essential to help some improvement in pronunciation.

In giving the segmental drill can be done in such an enjoyable approach for instance the usage of segmental drilling of minimal pairs. Drilling minimal pairs can be in form game, such as Chinese whispers (Junya and Meyer, 2000). The practical implementation can be done by setting the students into two rows. Each of the rows whispers a sentence that consist a minimal pairs or more. The last person in each row will retell the intended sentences. Kelly (2000) suggests Phonemic Crossword can be used as an active drilling practice. In the classroom the students are assigned to complete the missing sounds instead of the letters for the crossword in pairs. By doing such activity students are also able to practice to pronounce the sound at the same time while they completing the sounds.

Jazz chant can also be use to teach segmental feature of English sounds by focusing on language form or structure (Graham, 2000). Here jazz chats can also be used for example by proving two samples of sounds ending form for the simple present and past tense sounds ( this is a controlled technique). Let the learner notices the sounds' changing of the verb form used. For practice set (the free technique), the classroom practice can be in form of assigning the class into pairs activity to make their own jazz chants pattern which focus of the verb sounds changes, after that let the pairs to present in front of the class.

Tongue twisters can be used as one the techniques to teach English fluency. Tongue twister for teaching pronunciation was introduced by Peter Piper in his book Peter Piper's practical principles of plain and perfect pronunciation (1970). Tongue twister technique is good to be used in helping ESL/EFL students in pronouncing and announcing the difficult English sounds and to help them to develop clarity of speech. For the purpose of improving students' pronunciation, instead of pronouncing the tongue twister in fast pace, it is important to read it slowly and correctly, so the alliteration of each sound can be notice by the learners. The practical implementation of this technique can be done either in the traditional way, which is in a choral reciting or can be in more competitive setting. Tongue twisters can also be presented through game. The game can be played by throwing a soft item to the intended student within small group, and then the student who is chosen must recite the tongue twister. The winner is the group which can pronounce the more correct tongue twisters.

Further discussion as spoken features of the non-natives speaker are typically noticeable and may affect the intelligibility. The discussion tries to relate 
little bit of the current condition of the learners to the issue of intelligibility. To respond to condition of the participants, Jenkins (2002) suggests emphasizing the intelligibility and variability of English dialect between interlocutors to minimize misunderstanding in communication. Thus, the element of teaching pronunciation that can be used in to modify the problematic segment of the participants is that students do not necessarily imitate an ideal type of speech used by a particular group of native speakers. For example, the usage of unstressed vowel (schwa) is not significantly taught as long as both speakers understand each other. In the same vein Gilbert (2009) believes if students can master the basic of English communication is accepted.

Finally despite those problematic segment produced by the learners of second semester Study Program of English all are improvable. The success of teaching pronunciation is not merely seen from one side of view but should be seen from many angles. As Dickerson (2010) also suggests that in teaching pronunciation, teacher should focus on the articulation of sounds and teach segmental and suprasegmental

\section{Bibliography}

Acton, B. (2011). Essential, HapticallyIntegrated English Pronunciation (EHIEP). Instruction's manual.

Trinity Western University. Available at: Acton-2011EHIEP_intro.pdf

Budden, J. (2011). Using Poetry. Retrieved October 16th, 2012 from http://www.teachingenglish.org.uk/ language-assistant/teachingtips/using-poetry

Burston, J. (2005). Video dubbing projects in the foreign language curriculum. CALICO Journal. Vol.23 no.1. Retrieved October 16th, 2012 from calico.org

Chiu, Y. (2012). Can film dubbing projects facilitate EFL learner's acquisition of English pronunciation?.

British Journal of Educational Technology. Vol.43. Issue 1. features in integrated methods which possible to multi-modalities exposure.

\section{Conclusion}

This research has revealed that the second semester students of The Study Program of English Universitas Brawijaya have typical segmental features which are different from that of native speaker. The typical segmental features were detected through voice spectrogram software namely PRAAT. The segmental characteristic features of the second semester students were presented in formant: the spectral peaks of the sound spectrum (Gunnar Fant (1960). Those segmental features were characterized by the first formant (F1) and the second formant (F2). Finally the typical segmental features of the second semester students of Study Program of English Universitas Brawijaya were given modification to improve the accuracy in pronunciation. Some propossed techniques of teaching segmental were drilling, teaching minimal pairs, chanting with jazz chant and rhyming with tongue twisters.

Retrieved October, 16th, 2012 from onlinelibrary.wiley.com

Dickerson, W. B. (2011). Upstream destressing: Another step toward natural speech. In. J. Levis \& K. LeVelle (Eds.). Proceedings of the 2nd Pronunciation in Second Language Learning and Teaching Conference, Sept. 2010. (pp. 7081), Ames, IA: Iowa State University. Available at: pslltconference.com/index.php

Dickerson, W.B. (2010). Walking the walk: integrating the story of English phonology. In J.M Levis \& K. LeVelle (Eds), Proceeding of the 1st Pronunciation in Second Language Learning and Teaching Conference (pp. (-23), Ames, IA: Iowa State University. Available at: http://apling.public.iastate.edu/PSL LT/2009/2009pslltproceedings.pdf 
Gilbert, J. (2008). Teaching pronunciation: Using the prosody pyramid. Available at: http://www.cambrige.org/otherfiles/ downloads/esl/booklets/GilbertTaeching- Pronunciation.pdf Graham, C. (2000). Jazz Chants Old and New: student book. Oxford University Press.

Harmer, J. (2007). The practice of English language teaching (4th.ed). Harlow. England. Pearson Education

Jenkins, J. (2002). A sociolinguistically based, empirically researched pronunciation syllabus for English as an international language. Applied Linguistics, 23(1), 83-103.

Joaquin, A.D. (2009). "Drill, baby, drill": Exploring a Neurobiological Basis for the Teaching of Segmentals in the ESL/EFL Classroom. Teachers Articles: volume 39 article 2. Retrieved October 16th,2012 from http://www.asianefljournal.com/pta_October_2009_aj. php

Junya, A \& Meyer,L. (2000). Pronunciation Training. Retrieved October16,
2012 from http://www.cenglish.com/files/scans/06myc_pro nunciation_lmeyer.pdf

Kelly, G. (2000). How to teach pronunciation. London: Longman.

Macaro, E. (2001). Learning Strategies in foreign and second language classroom. New York. Continuum.

Mathew, I. (2005). Errors in pronunciation by learners of English as foreign language whose first language are Indonesia, Gayo and Acehnese. MONASH University Linguistic paper 2005. Vol.3 number 2. Retrieved October 12, 2012 from http://arrow.monash.edu.au/vital/ac cess/

Matthews, C. (1994). Speaking solution. London: Longman

Thompson, S.,Taylor, K., \& Gray, H. (2001). Pronunciation with an eye on multiple intelligences. WATESOL convention. Fall 2001. Retrived November 252014 from http://www.soundsofenglish.org/Pr esentations/WATESOL2001/multip leintelligencesactivities.htm

Tsui, A.B.M. (1994). English conversation. Oxford University Press 